P586

\title{
Left ventricular volume and mass adaptation after 58-days head-down bed-rest assessed by cine-MRI, and effectiveness of high-intensity jump training countermeasure
}

\author{
R. Egoriti ${ }^{1}$, F. Landreani ${ }^{1}$, L. Costantini ${ }^{2}$, E. Mulder ${ }^{3}$, D. Gerlach ${ }^{3}$, P. Vaida ${ }^{4}$, P.-F. Migeotte ${ }^{5}$, E.G. Caiani ${ }^{1}$
}

${ }^{1}$ Politecnico di Milano, Milan, Italy; ${ }^{2}$ Azienda Sanitaria Locale Lecce - P.O. Santa Caterina Novella, U.O. di Cardiologia e UTIC, Lecce, Italy; ${ }^{3}$ Deutsches Zentrum für Luft- und Raumfahrt e.V. (DLR), Institute of Aerospace Medicine, Space Physiology, Koln, Germany; ${ }^{4}$ University of Bordeaux, ENSCognitique, Bordeaux, France; ${ }^{5}$ Université libre de Bruxelles (ULB), Brussels, Belgium

Funding Acknowledgement: Italian Space Agency (contract 2018-7-U.0),CNES/DAR 4800000965,BELSPO, via the European Space Agency PRODEX program (PEA 4000110826)

Aims: Prolonged immobilization generates cardiac deconditioning, a risk factor for cardiovascular disease, and efficient countermeasures (CM) are needed to prevent it. Our aim was to assess by Cine-MRI the effects of long-term strict head-down ( -6 degrees) bed-rest (BR) deconditioning, and the effectiveness of high-intensity jump training CM, on left ventricular (LV) function and mass.

Methods: 23 male participants ( $29 \pm 6$ years, $181 \pm 6 \mathrm{~cm}, 77 \pm 7 \mathrm{~kg}$ ) were enrolled. The experiment was conducted at: envihab (Koln, DLR, Germany) as part of the European Space Agency BR studies. Volunteers were randomly allocated to the jump training group (JUMP, $n=12$ ) or the control group (CTRL, $n=11$ ). A typical training session consisted of $4 \times 10$ countermovement jumps and $2 \times 10$ hops in a sledge jump system, with 5-6 sessions per week.

Steady-state free precession cine-MRI images were obtained (25 frames/cardiac cycle, $8 \mathrm{~mm}$ thickness, no gap, no overlap) as stack of shortaxis images covering the whole LV from base to apex, before (PRE) and after 58-days (HDT58) of BR. Endocardial and epicardial semi-automated contouring was performed using custom software.
Results: In CTRL group, at HDT58 a reduction in LV mass (9\%), enddiastolic (21\%), end-systolic ( $8 \%$ ) and stroke volume $(23 \%)$ were observed, while ejection fraction did not change. In JUMP group, the reduction in LV end-diastolic volume was only by $9 \%$, followed by a decrease in endsystolic (10\%) and stroke volume (10\%), with a preservation of LV mass. In both groups, ejection fraction did not change.

Conclusions: Cardiac adaptation to deconditioning due to immobilization induced by BR resulted in a reduction of cardiac volumes and function, together with a decrease in LV mass. Interestingly, the applied JUMP countermeasure appeared able to partially reverse these effects, in particular by reducing the decrease in end-diastolic volume and preserving LV mass. This information could be useful for better understanding physiologic changes in patients undergoing long periods of immobilization, as well as to apply the studied countermeasure during space flight to reduce cardiac deconditioning.

\begin{tabular}{|c|c|c|c|c|c|c|}
\hline & & EDV (ml) & ESV (ml) & $\mathrm{SV}(\mathrm{ml})$ & $\mathrm{EF}(\%)$ & Mass (g) \\
\hline \multirow[t]{2}{*}{ CTRL } & PRE & $171(163 ; 191)$ & $60(58 ; 65)$ & $113(102 ; 124)$ & $64(63 ; 67)$ & $133(121 ; 160)$ \\
\hline & HDT58 & $143(131 ; 149)^{*}$ & $56(50 ; 58)^{\star}$ & $86(83 ; 92)^{\star}$ & $62(60 ; 63)^{*}$ & $127(106 ; 138)^{\star}$ \\
\hline \multirow[t]{2}{*}{ JUMP } & PRE & $156(140 ; 204)$ & $70(61 ; 84)$ & $91(78 ; 117)$ & $58(56 ; 60)$ & $118(104 ; 134)$ \\
\hline & HDT58 & $141(135 ; 175)^{*}$ & $63(54 ; 69)^{*}$ & $90(78 ; 92)^{*}$ & $58(55 ; 60)$ & $113(105 ; 128)$ \\
\hline
\end{tabular}

Results expressed as median (25th; 75 th percentiles). ${ }^{*} \mathrm{p}<0.05$ Wilcoxon non parametric paired test (PRE vs HDT58).

CTRL: control group; JUMP: countermeasure group. 